\title{
Présentation
}

\section{Le groupe de travail sur la sécurité des contrôles radiographiques industriels}

Les contrôles non destructifs (CND) sont mis en œuvre dans pratiquement tous les domaines de l'industrie (pétrole, nucléaire, aéronautique, etc.). Ils constituent un élément clé de la sécurité et de la sûreté des installations, soit qu'il s'agisse de justifier de la qualité d'une installation neuve, soit de sa tenue dans le temps aux contraintes d'exploitation. Les contrôles non destructifs utilisent différentes techniques, les plus utilisées étant les ultrasons, les courants de Foucault, et la radiographie industrielle. C'est cette dernière technique qui a motivé le travail présenté dans ce numéro spécial de notre revue.

Le lecteur trouvera, notamment dans le chapitre «matériel », plus de détails sur la technique mise en œuvre. Pour résumer en quelques mots cette pratique : on utilise les rayonnements ionisants, associés à des films photographiques (argentiques ou numériques) pour détecter les défauts internes des matériaux, chaque exposition est appelée «tir ». Les contrôles sont réalisés soit à poste fixe dans les ateliers de fabrication des éléments, soit dans les installations industrielles. C'est bien sûr ce mode de travail qui présente le plus de risques. Ces rayonnements sont produits par des sources radioactives dans la très grande majorité des cas ou par des générateurs de rayons $X$. Les sources sont mises en œuvre en France dans des appareils appelés Gammagraphes ${ }^{\circledR}$ qui assurent les fonctions de conteneur de transport (type B-U) et de projecteur, à l'aide de gaines de télécommande et d'éjection. L'activité des sources est de l'ordre de 4,4 TBq de ${ }^{192} \operatorname{Ir}$ (radio nucléide le plus utilisé), les débits d'équivalent de dose sont très importants, avec pour ligne de défense, un système de balisage délimitant une zone d'exclusion dite « zone d'intervention ». L'équipe qui réalise ces contrôles est composée de deux intervenants, dont au moins un des deux a satisfait au contrôle attribuant le Certificat d'Aptitude à la Manipulation de Radiographie Industrielle (CAMARI). Lorsque ces contrôles ont lieu sur site, le travail est réalisé habituellement de nuit pour éviter les co-activités, mais ceci n'est pas toujours possible, notamment avec le personnel d'exploitation des installations.

Il y a donc deux populations à protéger : les intervenants non liés à l'intervention de contrôle, et les radiologues industriels eux-mêmes. En France, nous n'avons pas observé depuis plus de 20 ans d'accident d'irradiation aigu du fait de ces techniques, mais à l'étranger les accidents à déplorer se sont produits à la suite de pertes de sources.

Le travail réalisé dans le cadre de ce groupe initié par la Section de protection technique de la SFRP, auquel se sont joints dès sa création les professionnels de 
la Confédération Française des Essais Non Destructifs (COFREND) a débuté fin 2006 pour aboutir à une journée de présentation spécifique le 30 janvier 2008. Ce groupe n'est pas le premier à avoir travaillé sur le sujet, mais la particularité que nous revendiquons est de tenter de concilier les approches techniques, réglementaires mais aussi sociales, organisationnelles et humaines car il est apparu dès les premiers travaux produits notamment par l'Université de Bordeaux (A. Garrigou et collaborateurs) en collaboration avec EDF en 2005, que c'est cette approche globale qui peut permettre d'améliorer le niveau de sécurité des interventions, en mettant l'intervenant « radiologue industriel » au centre du processus de mise en œuvre de sources radioactives de forte activité en milieu industriel (fréquemment jusqu'à 4,4 TBq de ${ }^{192} \mathrm{Ir}$ ).

\section{Les principes qui ont guidé nos travaux ont été :}

- De réunir des acteurs du contrôle non destructif : les différentes spécialités ont été sollicitées, comme des professionnels (intervenants et managers), des donneurs d'ordre, des ergonomes, des médecins, des membres de l'administration, et bien sûr des radioprotectionnistes, soit un total de 60 personnes. Neuf ateliers ont été créés, animés chacun par un expert du domaine.

- De favoriser l'émergence d'idées en faisant exprimer l'ensemble des membres en leur nom propre, dans le cadre de la SFRP, ou de la COFREND, sans engagement de leur employeur ou administration. Dans le même objectif, l'unanimité n'a pas été recherchée dans la rédaction des synthèses, plusieurs points de vue peuvent être cités.

- Les documents produits ne constituent donc en aucun cas un « référentiel», mais simplement un " guide » qu'il appartient à chacun de s'approprier et de mettre en œuvre, dans le cadre de ses propres responsabilités. Les bonnes pratiques identifiées sont proposées, par exemple aux professionnels de la COFREND. Le premier principe de la radioprotection, la justification de l'activité, n'a donc pas été approfondi dans le cadre de ce GT. Sur ce principe, la COFREND continue à travailler avec l'Autorité de sûreté nucléaire (ASN), sur la substitution de la technique de radiographie par d'autres techniques, en tenant compte de nombreux autres paramètres qui entrent en ligne de compte comme la sûreté des installations, les nuisances autres générées, la faisabilité technique etc.

\section{L'intervenant au centre des réflexions}

L'intervenant radiologue industriel a en charge la mise en œuvre du contrôle dans un environnement souvent hostile nécessitant une adaptabilité importante, mais il 
a aussi en charge directe, sans autre ligne de défense que son attention, sa sécurité et celle des autres travailleurs en cas de co-activité. Le contexte organisationnel est aussi dégradé, du fait du travail de nuit, l'isolement des radiologues induisant une charge supplémentaire.

Nous avons donc partagé notre travail, à l'image d'un processus industriel, en quatre parties :

- Les « contraintes » constituées principalement par la réglementation du travail et les codes de transport à respecter. Ces ateliers ont produit leur travail en parallèle de la nouvelle rédaction des textes décrivant la pratique.

- Les « supports» ont été traités par les ateliers « formation» et « matériel». Les textes décrivant la formation ont aussi évolué en parallèle des travaux $\mathrm{du}$ GT. Les propositions de matériel innovant visent à constituer de nouvelles lignes de défense passive, sans augmenter la charge de travail des intervenants.

- Des études spécifiques visent à aider les acteurs dans la démarche de prévention des risques intégrés à l'activité. Ces études concernent la dosimétrie de l'intervenant, une aide à l'évaluation des risques basée sur une approche ergonomique qui constitue le point central de nos travaux, mais aussi, des outils qui ont fait leur preuve dans l'amélioration de la sûreté des installations nucléaires et qui sont transposables à la radiographie industrielle : la répartition des responsabilités et un guide d'auto-évaluation. Le principe de la description des responsabilités est de dissocier l'acteur qui réalise l'action de celui qui en porte la responsabilité juridique principale : on peut sous-traiter une action, cela ne signifie pas que l'on se décharge de la responsabilité afférente. Cet atelier a utilisé les travaux des GT «PACA » et « Normandie » qui constituent une description détaillée de la pratique. Le guide d'auto-évaluation, lui, doit permettre à chacun, en dehors d'un contexte d'inspection, d'améliorer la maîtrise de la mise en œuvre des contrôles radiographiques industriels. Ce type de démarche a fait ses preuves dans les domaines de la sûreté nucléaire, plus récemment de la radioprotection, il doit aider les responsables à sécuriser et optimiser la pratique.

- Le retour d'expérience. Là aussi, c'est l'analyse des écarts qui a été un levier fort de l'amélioration de la sûreté nucléaire; cette analyse des signaux faibles dans le domaine de la radioprotection constitue une clé à l'évolution indispensable de la radioprotection, accompagnant l'évolution des niveaux d'exigence et de transparence. La base de données RELIR existait et sera utilisée, ce qui assurera une pérennité indispensable au retour d'expérience. 
Les neuf ateliers qui ont été constitués sont :

\begin{tabular}{cll}
\hline $\mathbf{N}^{\circ}$ & \multicolumn{1}{c}{ Atelier } & \multicolumn{1}{c}{ Animateur(s) } \\
\hline 1 & Le référentiel réglementaire & Olivier Couasnon \\
\hline 2 & Le retour d'expérience & Christian Lefaure et Céline Bataille \\
\hline 3 & La formation & Alain Biau \\
\hline 4 & Les matériels et leur gestion & Frederic Fournet \\
\hline 5 & La dosimétrie & François Coletti et Didier Paul \\
6 & Les études de poste & Bernard Le Guen \\
\hline 7 & Le guide d'auto-évaluation & Philippe Roiné \\
\hline 8 & Les limites de responsabilités entre les entreprises & Jean Gonnet \\
\hline 9 & Le transport & Alain Laumond \\
\hline
\end{tabular}

\section{La forme de la publication}

Pour ce numéro supplémentaire de la revue «Radioprotection», plutôt que le respect strict des références bibliographiques particulièrement pour les normes et textes, nous avons choisi de favoriser la facilité de lecture en maintenant ces références intégrées aux textes.

\section{Conclusion}

Ce travail constitue un outil de la prévention, et un point étape de la pratique du contrôle radiographique industriel, et nous le souhaitons, dans l'évolution de la radioprotection, allant vers l'intégration accrue des aspects sociaux, organisationnels et humains.

Pour le futur, il appartient à chaque acteur du processus de pérenniser ce travail pour permettre à tous, $\mathrm{y}$ compris les industriels travaillant dans des secteurs très divers, d'avoir un niveau d'exigence identique, en leur fournissant les outils indispensables à l'exercice de leurs responsabilités.

\section{Remerciements}

Il ne m'est pas possible de remercier individuellement ici les soixante membres du GT, cités dans les publications de chaque atelier, ni les animateurs des neuf ateliers qui ont souvent pris sur leur temps personnel pour l'organisation des réunions et la rédaction des synthèses des ateliers. Qu'ils sachent tous que la SFRP les remercie vivement. 
À titre personnel, je tiens à exprimer ma reconnaissance à Christian Lefaure qui a initié ce travail à travers la création d'un groupe similaire auquel il a participé dans le cadre de la Commission Européenne.

Mes remerciements vont aussi à Alain Biau, qui après avoir aidé et soutenu pendant de nombreuses années les radioprotectionnistes opérationnels français, il nous a également montré qu'une radioprotection n'est efficace et n'a de sens qu'en intégrant l'aspect humain des événements. Il a été l'acteur principal de ces travaux.

G. Abéla 